\title{
$\lambda$-POWER INTEGRALS ON THE CANTOR TYPE SETS
}

\author{
SHUSHANG FU \\ (Communicated by Andrew M. Bruckner) \\ Dedicated to Professor R. Darst on the occasion of his sixtieth birthday
}

\begin{abstract}
We introduce the notions of $\lambda$-power dyadic derivatives and $\lambda$ power dyadic integrals, so that, in particular, the Cantor ternery function is an indefinite integral of its derivative. Furthermore, under certain conditions on the integrands we can give a Riemann-type definition to the $\lambda$-power dyadic integral.
\end{abstract}

\section{INTRODUCTION}

One of the basic problems of analysis is to find a notion of integration such that integration and differentiation are inverse operators for a much larger class of functions. However, in accordance with contemporary notions of integration, the integral of a trivial function, which equals zero almost everywhere, is zero. Therefore, there is no notion of integration such that a singular function represents an indefinite integral of its derivative, since the derivative of a singular function is a trivial function.

The main motivation of the paper is to find the notions of differentiation and integration such that some singular functions are the definite integrals of their derivatives. The reason why the differential and integral in the ordinary sense do not work for the class of singular functions is that the set $\left\{x: f^{\prime}(x)=\right.$ $\left.-\infty, \bar{f}^{\prime}(x)=+\infty\right\}$ has an uncountable order, that is, there are too many points at which the change in function is much faster than the change in variable. This gives us a hint how to solve the problem.

We replace the difference quotient $\frac{\Delta y}{\Delta x}$ by $\frac{\Delta y}{|\Delta x|^{\lambda}}, 0<\lambda<1$, and define the $\lambda$-power derivative of $f$ at $x$ by $f_{\lambda-p}^{\prime}(x)=\lim _{\Delta x \rightarrow 0} \frac{\Delta y}{|\Delta x|^{\lambda}}$. This is called Lipschitz numbers by Besicovish and has been studied by Thomson [5]. Clearly, if the ordinary derivative of $f$ exists, then its $\lambda$-power derivative is infinite. But in some cases where the ordinary derivative of $f$ is infinite, the $\lambda$-power derivative of $f$ is finite. The latter situation is what we want. Note that the $\lambda$ power derivative cannot be applied to a singular function, because the $\lambda$-power

Received by the editors October 8, 1993 and, in revised form, January 21, 1994.

1991 Mathematics Subject Classification. Primary 26A39; Secondary 28A75.

Key words and phrases. Singular function, Cantor type set, Hausdorff dimension and Hausdorff measure, $\lambda$-power dyadic derivative, $\lambda$-power dyadic integral, $\lambda$-power Riemann integral. 
derivative may not exist at most points. For example, consider the Cantor ternary function.

We modify the $\lambda$-power derivative to get the concept of the $\lambda$-power dyadic derivative which will work for our purpose on the special sets of measure zero. The ideal of the dyadic derivative is derived from the papers by Kahane [2], Pacquement [4] and Gordon [1]. Since we deal with the thin set, we will use the Hausdorff measure instead of the Lebesque measure.

In our exposition, we first describe the Cantor type sets which will be used to define the generalized differential and integral. We next introduce and study the $\lambda$-power dyadic derivative and $\lambda$-power dyadic integral. Finally, we define the $\lambda$-power Riemann integral and show that the $\lambda$-PR integral and $\lambda$-PD integral are equivalent for the continuous integrands.

\section{2. $\lambda$-POWER DYADIC DERIVATIVE}

Let $[a, b]$ be a closed bounded interval. A partition of $[a, b]$ is given by

$$
a=a_{1}^{1}<b_{1}^{1}<a_{1}^{2}<\cdots<b_{1}^{n}=b .
$$

Write $U_{1}=\bigcup_{j=1}^{n} U_{1}^{j}$, where $U_{1}^{j}=\left[a_{1}^{j}, b_{1}^{j}\right], j=1,2, \ldots, n ; V_{1}=\bigcup_{j=1}^{n-1} V_{1}^{j}$, where $V_{1}^{j}=\left(b_{1}^{j}, a_{1}^{j+1}\right), j=1,2, \ldots, n-1$. A partition of $U_{1}^{j}, j=1,2, \ldots$, $n$, is given by

$$
a_{1}^{j}=a_{2}^{(j-1) n+1}<b_{2}^{(j-1) n+1}<a_{2}^{(j-1) n+2}<\cdots<b_{1}^{j n}=b_{1}^{j} .
$$

Write $U_{2}=\bigcup_{j=1}^{n^{2}} U_{2}^{j}$, where $U_{2}^{j}=\left[a_{2}^{j}, b_{2}^{j}\right], j=1,2, \ldots, n^{2}$, and write $V_{2}=\bigcup_{j=1}^{n(n-1)} V_{2}^{j}$, where $V_{2}^{j}=\left(b_{2}^{j}, a_{2}^{j+1}\right), j=1,2, \ldots, n(n-1)$.

Continue this process to obtain a sequence of intervals $U_{m}=\left\{U_{m}^{j}\right\}_{j=1}^{n^{m}}$ and $V_{m}=\left\{V_{m}^{j}\right\}_{j=1}^{n(n-1)}$. Define $C=\bigcap_{m=1}^{\infty} U_{m}=[a, b] \backslash \bigcup_{m=1}^{\infty} V_{m}$. Then $C$ is a perfect set. A perfect set $C$ is a Cantor type if $m C=0, H-\operatorname{dim} C=\lambda, 0<$ $H^{\lambda}(C)<\infty$ and $H^{\lambda}\left(C \bigcap\left(a_{m}^{j(m)}, b_{m}^{j(m)}\right)\right)=\left(b_{m}^{j(m)}-a_{m}^{j(m)}\right)^{\lambda}$, where $m$ denotes the Lebesque measure, $H$-dim the Hausdorff dimension and $H^{\lambda}$ the Hausdorff measure.

Let $C$ be a Cantor type set, and let $H-\operatorname{dim} C=\lambda$. Let $D$ represent the set of endpoints of intervals $U_{m}^{j}, m=1,2, \ldots, i=1,2, \ldots, n^{m}$.

Definition 1. Let $F:[a, b] \rightarrow R$, and let $x \in C \backslash D$ and $x=\bigcap_{m=1}^{\infty}\left(a_{m}^{j(m)}, b_{m}^{j(m)}\right)$. The function $F$ is $\lambda$-power dyadic differentiable at $x$ if the limit

$$
\lim _{n \rightarrow \infty} \frac{F\left(b_{m}^{j(m)}\right)-F\left(a_{m}^{j(m)}\right)}{\left(b_{m}^{j(m)}-a_{m}^{j(m)}\right)^{\lambda}}
$$

exists. We will use $F_{\lambda \text {-PD }}^{\prime}(x)$ to denote the $\lambda$-power dyadic derivative at $x$.

Here are several obvious statements. If $F$ is $\lambda$-PD differentiable at $x$ and $\alpha$ is a real number, then $(\alpha F(x))_{\lambda \text {-PD }}^{\prime}=\alpha F_{\lambda \text {-PD }}^{\prime}(x)$. If $F$ and $G$ are $\lambda$-PD differentiable at $x$, then the sum $F+G$ is $\lambda$-PD differentiable at $x$ and $(F(x)+G(x))_{\lambda-\mathrm{PD}}^{\prime}=F_{\lambda-\mathrm{PD}}^{\prime}(x)+G_{\lambda-\mathrm{PD}}^{\prime}(x)$.

Example. Let $f$ be the Cantor ternary function on $[0,1]$. Let $x \in C \backslash D$ and $x=\bigcap_{m=1}^{\infty}\left(a_{m}^{j(m)}, b_{m}^{j(m)}\right)$. 
Compute

$$
\frac{f\left(b_{m}^{j(m)}\right)-f\left(a_{m}^{j(m)}\right)}{\left(b_{m}^{j(m)}-a_{m}^{j(m)}\right)^{\lambda}}=\frac{\frac{1}{2^{m}}}{\frac{1}{3^{m \lambda}}}=\left(\frac{3^{\lambda}}{2}\right)^{m}=1
$$

where $\lambda=\frac{\log 2}{\log 3}$ is the Hausdorff dimension of the Cantor set $C$. This shows that $f_{\lambda-\mathrm{PD}}(x)=1$ on $C \backslash D$.

\section{3. $\lambda$-POWER DYADIC INTEGRAL}

We will give a descriptive definition of the $\lambda$-power dyadic integral. First, we recall some terminology. Let $[a, b]$ be a bounded closed interval. A finite set of pairs $\left\{\left[u_{i}, v_{i}\right], x_{i}\right\}_{i=1}^{I}$ is an allotted partition on $[a, b]$ if $\left\{\left[u_{i}, v_{i}\right]\right\}_{i=1}^{I}$ is a partition of the interval $[a, b]$. We call $x_{i}$ the associated point of the pair $\left\{\left[u_{i}, v_{i}\right], x_{i}\right\}$. Let $\delta(x)$ be a positive function defined on $[a, b]$. The allotted partition $\left\{\left[u_{i}, v_{i}\right], x_{i}\right\}_{i=1}^{I}$ is $\delta$-fine if $x_{i} \in\left[u_{i}, v_{i}\right]$ and $\left[u_{i}, v_{i}\right] \subseteq B\left(x_{i}, \delta\left(x_{i}\right)\right)$, where $B\left(x_{i}, \delta\left(x_{i}\right)\right)$ denotes an open ball.

Theorem 1. Let $F(x)$ be a continuous function on $[a, b]$. If $F^{\prime}(x) \geq 0$ on $[a, b] \backslash C$ and $F_{\lambda-\mathrm{PD}}^{\prime}(x) \geq 0$ nearly everywhere (except on a countable set) on $C \backslash D$, then $F$ is monotone non-decreasing on $[a, b]$.

Proof. Let $E=\left\{x \in C \backslash D: \nexists F_{\lambda-\mathrm{PD}}^{\prime}(x)\right\}$ or $\left.F_{\lambda \text {-PD }}^{\prime}(x)<0\right\}$. Then $E$ is countable. Given $\varepsilon>0$ and $\xi \in C \backslash(D \cup E)$, since $F(x)$ is $\lambda$-PD differentiable at $\xi$, we can take an integral $I_{\xi}=\left(a_{m}^{j(m)}, b_{m}^{j(m)}\right)$ such that

$$
\left|F\left(b_{m}^{j(m)}\right)-F\left(a_{m}^{j(m)}\right)-F_{\lambda-\mathrm{PD}}^{\prime}(\xi)\left(b_{m}^{j(m)}-a_{m}^{j(m)}\right)^{\lambda}\right|<\varepsilon\left(b_{m}^{j(m)}-a_{m}^{j(m)}\right)^{\lambda}
$$

where $a_{m}^{j(m)}<\xi<b_{m}^{j(m)}$.

Let $D \cup E=\left\{\theta_{i}\right\}_{i=1}^{\infty}$. From the continuity of $F$, for each $\theta_{i}$, there exists an interval $I_{\theta_{i}}=\left(\theta_{i}-\eta_{i}, \theta_{i}+\eta_{i}\right)$ such that

$$
\left|F\left(x_{2}\right)-F\left(x_{1}\right)\right|<\frac{\varepsilon}{2^{i}}
$$

where $x_{1}, x_{2} \in\left[\theta_{i}-\eta_{i}, \theta_{i}+\eta_{i}\right]$.

Since $C \subseteq \bigcup\left\{I_{\xi}: \xi \in C\right\}$, we may apply the Heine-Borel theorem, so there is a finite subcover $A=\left\{I_{\xi^{j}}\right\}_{j=1}^{J}$. We divide the intervals $I_{\xi^{j}}, j=1,2, \ldots, J$, into two groups. We shall say that an interval $I_{\xi^{j}}$ is of the first class if the associated point $\xi^{j} \in C \backslash(D \cup E), I_{\xi^{j}}=\left(a_{m_{j}}^{j\left(m_{j}\right)}, b_{m_{j}}^{j\left(m_{j}\right)}\right)$, and otherwise of the second class, i.e, $\xi^{j} \in D \cup E, I_{\xi^{j}}=\left(\theta_{i(j)}-\eta_{i(j)}, \theta_{i(j)}+\eta_{i(j)}\right)$. Clearly, if first class intervals $\left(a_{n}^{j(n)}, b_{n}^{j(n)}\right) \cap\left(a_{m}^{j(m)}, b_{m}^{j(m)}\right) \neq \varnothing$, then $\left(a_{n}^{j(n)}, b_{n}^{j(n)}\right) \subseteq\left(a_{m}^{j(m)}, b_{m}^{j(m)}\right)$ or $\left(a_{m}^{j(m)}, b_{m}^{j(m)}\right) \subseteq\left(a_{n}^{j(n)}, b_{n}^{j(n)}\right)$. Therefore, without loss of generality we may suppose that the first class intervals in $A$ are pairwise disjoint.

Consider the union of elements of $A, U=\bigcup_{j=1}^{J} I_{\xi^{j}}=\bigcup_{j=1}^{P}\left(a_{j}, b_{j}\right)$, where intervals $\left(a_{j}, b_{j}\right)$ are pairwise disjoint. Note that $\left(a_{j}, b_{j}\right), j=1,2 \ldots, P$, is of the finite union of elements of $A$, that is, $\left(a_{j}, b_{j}\right)=\bigcup_{t=1}^{T} I_{\xi^{i(t)}}, I_{\xi^{i(t)}} \in$ $A$. If necessary discard some intervals $I_{\xi^{(t)}}$; we write $I_{\xi^{i(t)}}=\left(c_{t}, d_{t}\right), t=$ $1,2, \ldots, T$, such that

$$
a_{j}=c_{1}<c_{2}<d_{1}<c_{3}<d_{2}<\cdots<d_{T}=b_{j} .
$$

We recall that the first class intervals are pairwise disjoint. Hence, there is a partition of $\left(a_{j}, b_{j}\right)$ such that the subinterval is either a first class interval of $A$ 
or a subinterval of a second class interval of $A$. Thus, there exists a partition $D_{1}$ of $U$ which consists of the first class intervals and the subintervals of the second class intervals.

Let $V-[a, b] \backslash U$. Then $V$ is a finite union of the closed intervals and $V \subset[a, b] \backslash C$. Since $F$ is differentiable on $[a, b] \backslash C$, for each $\xi$ in $[a, b] \backslash U$ there exists a $\delta(\xi)>0$ such that

$$
\left|F\left(x_{2}\right)-F\left(x_{1}\right)-F^{\prime}(\xi)\left(x_{2}-x_{1}\right)\right|<\varepsilon\left|x_{2}-x_{1}\right|
$$

where $\left[x_{1}, x_{2}\right] \subseteq B(\xi, \delta(\xi))$.

It is well known that for every positive function $\delta(x)$ defined on an interval $[\alpha, \beta]$, there is a $\delta$-fine division $D$ on $[\alpha, \beta]$ [3, p. 4]. Applying this theorem to each closed interval of $V$, we get a partition $D_{2}$ of $V$. We call a subinterval of the partition $D_{2}$ a third class interval. Let $D$ be the partition formed by the points in both $D_{1}$ and $D_{2}$. Relabelling the partition $D$, we write

$$
a=a_{0}<a_{1}<\cdots<a_{m}=b
$$

and $[a, b]=\bigcup_{i=0}^{m-1}\left[a_{i}, a_{i+1}\right]$. We obtain

$$
F(b)-F(a)=\sum_{i=0}^{m-1}\left(F\left(a_{i+1}\right)-F\left(a_{i}\right)\right)=\sum_{1}+\sum_{2}+\sum_{3}
$$

where $\sum_{i}$ denotes the partial sum of $\sum$ for which the interval $\left[a_{i}, a_{i+1}\right]$ is an $i$-class interval, $i=1,2,3$.

Consider first the term $\sum_{1}$. By (A),

$$
F\left(b_{n}^{j(n)}\right)-F\left(a_{n}^{j(n)}\right) \geq F_{\lambda-P D}^{\prime}(\xi)\left(b_{n}^{j(n)}-a_{n}^{j(n)}\right)^{\lambda}-\varepsilon\left(b_{n}^{j(n)}-a_{n}^{j(n)}\right)^{\lambda} .
$$

From this and $F_{\lambda \text {-PD }}^{\prime}(\xi) \geq 0$, we obtain

$$
F\left(b_{n}^{j(n)}\right)-F\left(a_{n}^{j(n)}\right) \geq-\varepsilon\left(b_{n}^{j(n)}-a_{n}^{j(n)}\right)^{\lambda} .
$$

By definition of the Cantor type set, we deduce

$$
F\left(b_{n}^{j(n)}\right)-F\left(a_{n}^{j(n)}\right) \geq-\varepsilon H^{\lambda}\left(\bigcap\left(a_{n}^{j(n)}, b_{n}^{j(n)}\right)\right) .
$$

Consequently, we have

$$
\sum_{1} \geq-\varepsilon \sum H^{\lambda}\left(C \cap\left(a_{n}^{j(n)}, b_{n}^{j(n)}\right)\right),
$$

since Hausdorff measure is an outer measure in the sense of Caratheodory. Now, by $(\mathrm{C})$

$$
\sum_{1} \geq-\varepsilon H^{\lambda}\left(C \cap\left(\bigcup\left(a_{n}^{j(n)}, b_{n}^{j(n)}\right)\right)\right) \geq-\varepsilon H^{\lambda}(C)
$$

It is routine to show the inequalities

$$
\sum_{2} \geq-\varepsilon \text { and } \sum_{3} \geq-\varepsilon(b-a)
$$

By (D) and (E),

$$
F(b)-F(a) \geq-\varepsilon\left(1+H^{\lambda}(C)+b-a\right) .
$$

Because of $\varepsilon$ arbitrary small, we get

$$
F(b) \geq F(a) .
$$


For each subinterval $[\alpha, \beta]$ of $[a, b]$, the same argument gives that

$$
F(\beta) \geq F(\alpha) \text {. }
$$

This shows that $F$ is monotone nondecreasing on $[a, b]$.

Corollary. Let $F$ be a continuous function on $[a, b]$. If $F^{\prime}(x)=0$ on $[a, b] \backslash C$ and $F_{\lambda-\mathrm{PD}}^{\prime}(x)=0$ n.e. on $C \backslash D$, then $F$ is constant.

We call $f$ a trivial function on $C$ if $f$ vanishes on $[a, b] \backslash C$.

Definition 2. A trivial function $f(x)$ on $C$ is said to be $\lambda$-power dyadic integrable on $[a, b]$ if there exists a continuous function $F(x)$ such that

$$
\begin{gathered}
F^{\prime}(x)=f(x)=0 \quad \text { on }[a, b] \backslash C, \\
F_{\lambda-\mathrm{PD}}^{\prime}(x)=f(x) \quad \text { nearly everywhere on } C \backslash D
\end{gathered}
$$

where $F_{\lambda \text {-PD }}^{\prime}(x)$ is the $\lambda$-power dyadic derivative of $F(x)$. The function $F(x)$ is called an indefinite integral of $f(x)$, and the definite integral on $[a, b]$, denoted by $\left(\lambda\right.$-PD) $\int_{a}^{b} f$, is defined as $F(b)-F(a)$,

$$
F(b)-F(a)=(\lambda-\mathrm{PD}) \int_{a}^{b} f .
$$

Example. Let $f$ be the Cantor ternary function defined on $[0,1]$. Then

$$
f(1)-f(0)=(\lambda-\mathrm{PD}) \int_{0}^{1} f_{\lambda-\mathrm{PD}}^{\prime}=(\lambda-\mathrm{PD}) \int_{0}^{1} 1 .
$$

The $\lambda$-PD integral has the usual properties of integral,

$$
\begin{gathered}
(\lambda-\mathrm{PD}) \int_{a}^{b}\left(C_{1} f_{1}+c_{2} f\right)=C_{1}(\lambda-\mathrm{PD}) \int_{a}^{b} f_{1}+C_{2}(\lambda-\mathrm{PD}) \int_{a}^{b} f_{2}, \\
(\lambda-\mathrm{PD}) \int_{a}^{b} f=(\lambda-\mathrm{PD}) \int_{a}^{c} f+(\lambda-\mathrm{PD}) \int_{c}^{b} f .
\end{gathered}
$$

Remark 1. It is interesting to compare the Lebesgue integral and the $\lambda$-PD integral. For example, if $f$ is $L$-integrable and $g=f$ a.e. on $[a, b]$, then $g$ is $L$-integrable and $(L) \int_{a}^{b} f=(L) \int_{a}^{b} g$. However, if $f$ is $\lambda$-PD integrable and $g=f$ n.e. on $[a, b]$, then $g$ is $\lambda$-PD integrable and ( $\lambda$-PD) $\int_{a}^{b} g=$ $\left(\lambda\right.$-PD) $\int_{a}^{b} f$. That is, for the Lebesgue integral we can neglect a Lebesgue measure zero set; but for the $\lambda$-PD integral we only can neglect a countable set. This demonstrates the difference between the Lebesgue integral and the $\lambda$-PD integral.

\section{4. $\lambda$-POWER RIEMANN INTEGRAL}

In this section we introduce the concept of the $\lambda$-power Riemann integral. We next show that the $\lambda$-PR integral and the $\lambda$-PD integral are equivalent for continuous integrands. Let $C$ be a Cantor type set and $H-\operatorname{dim} C=\lambda$. A trivial function $f$ on $C$ is continuous if the restriction function $\left.f\right|_{C}$ is continuous, where $\left.f\right|_{C}$ denotes the function $f$ restricted on $C$. 
Definition. A trivial function $f$ on $C$ is said to be $\lambda$-power Riemann integrable, if

$$
\sup _{\varepsilon} \inf _{U}\left\{\sum_{i=1}^{\infty} f\left(\xi_{i}\right)\left(b_{i}-a_{i}\right)^{\lambda}: C \subset U=\bigcup\left(a_{i}, b_{i}\right), \xi_{i} \in\left(a_{i}, b_{i}\right) \cap C, b_{i}-c_{i}<\varepsilon\right\}
$$

is finite.

Since $H^{\lambda}(C)<\infty$, a lower bounded trivial function $f$ is $\lambda$-PR integrable. We will be concerned with continuous integrands. We list the properties of the $\lambda$-PR integral, some of which will be used later.

(1) $(\lambda$-PR $) \int_{a}^{b} \alpha f+\beta g=\alpha(\lambda$-PR $) \int_{a}^{b} f+\beta(\lambda$-PR $) \int_{a}^{b} g$.

(2) $\left(\lambda\right.$-PR) $\int_{a}^{b} f=\left(\lambda\right.$-PR) $\int_{a}^{c} f+(\lambda$-PR $) \int_{c}^{b} f, a<c<b$.

(3) If $f=0$, nearly everywhere on $C$, then ( $\lambda$-PR) $\int_{a}^{b} f=0$.

(4) If $f \leq g$ nearly everywhere on $C$, then $\left(\lambda\right.$-PR) $\int_{a}^{b} f \leq\left(\lambda\right.$-PR) $\int_{a}^{b} g$.

(5) ( $\lambda$-PR) $\int_{a}^{b} 1=H^{\lambda}(C \cap[a, b])$.

Let $f$ be a continuous trivial function on $C$, and let $F(x)=(\lambda$-PR $) \int_{a}^{x} f$.

Theorem 2. Let $f(x)$ be a continuous trivial function on $C$. Then $F(x)$ is continuous on $[a, b], F^{\prime}(x)=0$ on $[a, b] \backslash C$ and $f_{\lambda-\mathrm{PD}}^{\prime}(x)=f(x)$ on $C \backslash D$. Proof. Let $[a, b] \backslash C=\bigcup_{i=1}^{\infty}\left(a_{i}, b_{i}\right)$. Clearly, for each $x$ in $\left(a_{i}, b_{i}\right)$, we have

$$
(\lambda \text {-PR }) \int_{a}^{x} f=(\lambda \text {-PR }) \int_{a}^{a_{i}} f .
$$

This shows that $F$ is constant on $\left(a_{i}, b_{i}\right)$ and $F^{\prime}(x)=0$ for $x \in\left(a_{i}, b_{i}\right)$. It also implies that $F$ is continuous on $\left(a_{i}, b_{i}\right)$.

We shall show the continuity of $F(x)$. For each $x \in C \backslash D$, let $x=$ $\bigcap_{n=1}^{\infty}\left(a_{n}^{j(n)}, b_{j}^{j(n)}\right)$ (the case when $x \in D$ is analogous). Given $\varepsilon>0$, from the continuity of $\left.f\right|_{C}$, there is a $\delta_{1}>0$ such that

$$
|f(y)-f(x)|<\varepsilon
$$

whenever $|y-x|<\delta_{1}$ and $y \in C$. Since $\lim _{n \rightarrow \infty}\left(b_{n}^{j(n)}-a_{n}^{j(n)}\right)=0$, there is an $n_{0}$ such that $\left|b_{n_{0}}^{j\left(n_{0}\right)}-a_{n_{0}}^{j\left(n_{0}\right)}\right|^{\lambda}<\frac{\varepsilon}{|f(x)|+\varepsilon}$. Let $\delta_{2}=\min \left(b_{n_{0}}^{j\left(n_{0}\right)}-x, x-a_{n_{0}}^{j\left(n_{0}\right)}\right)$, and let $\delta=\min \left(\delta_{1}, \delta_{2}\right)$. We have

$$
\begin{aligned}
|F(y)-F(x)| & \leq(\lambda \text {-PR }) \int_{x}^{y}(|f(x)|+\varepsilon) \\
& =(|f(x)|+\varepsilon) H^{\lambda}(C \cap[x, y]) \\
& \leq(|f(x)|+\varepsilon) H^{\lambda}\left(C \cap\left[a_{n_{0}}^{j\left(n_{0}\right)}, b_{n_{0}}^{j\left(n_{0}\right)}\right]\right) \\
& =(|f(x)|+\varepsilon)\left(b_{n_{0}}^{j\left(n_{0}\right)}-a_{n_{0}}^{j\left(n_{0}\right)}\right)^{\lambda}<\varepsilon
\end{aligned}
$$

whenever $|x-y|<\delta$.

We shall show that $F_{\lambda \text {-PD }}^{\prime}(x)=f(x)$ for $x \in C \backslash D$. Let

$$
x=\bigcap_{n=1}^{\infty}\left(a_{n}^{j(n)}, b_{n}^{j(n)}\right) .
$$


Given $\varepsilon>0$, there is a $\delta_{1}>0$ such that $|f(y)-f(x)|<\varepsilon$, whenever $|y-x|<$ $\delta_{1}$ and $y \in C$. Since $\lim _{n \rightarrow \infty}\left(b_{n}^{j(n)}-a_{n}^{j(n)}\right)=0$, there is an $n_{0}$ such that $\left|b_{n}^{j(n)}-a_{n}^{j(n)}\right|<\delta_{1}$ whenever $n>n_{0}$.

Consequently,

$$
\begin{aligned}
& \left|F\left(b_{n}^{j(n)}\right)-F\left(a_{n}^{j(n)}\right)-f(x)\left(b_{n}^{j(n)}-a_{n}^{j(n)}\right)^{\lambda}\right| \\
& \quad \leq \mid(\lambda \text {-PR }) \int_{a_{n}^{j(n)}}^{b_{n}^{j(n)}}(f(y)-f(x)) \mid \leq \varepsilon\left(b_{n}^{j(n)}-a_{n}^{j(n)}\right)^{\lambda} .
\end{aligned}
$$

That is, $\lim _{n \rightarrow \infty} \frac{F\left(b_{n}^{j(n)}\right)-F\left(a_{n}^{j(n)}\right)}{\left.b_{n}^{j(n)}-a_{n}^{j(n)}\right)^{\lambda}}=f(x)$.

Remark 2. From the theorem, a continuous trivial function $f$ on $C$ is $\lambda$-PD integrable and ( $\lambda$-PR) $\int_{a}^{b} f=\left(\lambda\right.$-PD) $\int_{a}^{b} f$. It is easy to show that if a trivial function $f$ on $C$ is $\lambda$-PD integrable and $\left.f\right|_{C}$ is continuous, then $f$ is $\lambda$-PR integrable and $\left(\lambda\right.$-PD) $\int_{a}^{b} f=(\lambda$-PR $) \int_{a}^{b} f$. Indeed, let $F(x)=\left(\lambda\right.$-PD) $\int_{a}^{x} f$ and $G(x)=(\lambda$-PR $) \int_{a}^{x} f$. By the above theorem, $G$ is the indefinite $\lambda$-PD integral and $G_{\lambda-\mathrm{PD}}^{\prime}(x)=f(x)$ for $x \in C \backslash D$. Since $F_{\lambda-\mathrm{PD}}^{\prime}(x)=f(x)$ n.e. on $C \backslash D$, we have $(F-G)_{\lambda \text {-PD }}^{\prime}=0$ n.e. on $C \backslash D$. This implies that $F-G=$ constant .

\section{ACKNOWLEDGMENT}

The author wishes to thank the referee for his valuable suggestions.

\section{REFERENCES}

1. R. Gordon, The inversion of approximate and dyadic derivatives using an extension of the Henstock integral, Real Anal. Exchange 16 (1990), 154-168.

2. J. Kahane, Une theorie de Denjoy des martingales dyadiques, Prepublication from Unite Associee CNRS757, Universite de Paris-Sud.

3. P. Y. Lee, Lanzhou lectures on Henstock integration, World Scientific Publishing, 1989.

4. A. Pacquenaent, Determination d'une fonction a moyen de sa derivee sur un resean binaire, C. R. Acad. Sci. Paris Ser. A 284 (1977), 365-368.

5. B. S. Thomson, Derivates of interval functions, Mem. Amer. Math. Soc. 93452 (1991). CHINA

Department of Mathematics, Fuzhou University, Fujian, 350002, People's Republic of

\section{CHNA}

